\title{
Arabidopsis PPP family of serine/threonine phosphatases
}

\section{Ilona Farkas ${ }^{1}$, Viktor Dombrádi ${ }^{1}$, Márton Miskei ${ }^{1,2}$, László Szabados ${ }^{3}$ and Csaba Koncz ${ }^{3,4}$}

\author{
${ }^{1}$ Department of Medical Chemistry, Research Center for Molecular Medicine, Medical and Health Science Center, University of \\ Debrecen, Egyetem tér 1, Debrecen H-4032, Hungary \\ ${ }^{2}$ Current address: Centre of Agricultural Sciences, Faculty of Agriculture, Department of Horticultural Sciences and Plant \\ Biotechnology, University of Debrecen, Egyetem tér 1, Debrecen H-4032, Hungary \\ ${ }^{3}$ Institute of Plant Biology, Biological Research Center of the Hungarian Academy of Sciences, Temesvári krt. 62, Szeged H-6726, \\ Hungary \\ ${ }^{4}$ Max-Planck-Institut für Züchtungsforschung, Carl-von-Linné-Weg 10, D-50829 Köln, Germany
}

\begin{abstract}
Serine/threonine-specific phosphoprotein phosphatases (PPPs) are ubiquitous enzymes in all eukaryotes, but their regulatory functions are largely unknown in higher plants. The Arabidopsis genome encodes 26 PPP catalytic subunits related to type 1, type $2 A$ and so-called novel phosphatases, including four plant-specific enzymes carrying large $\mathrm{N}$-terminal kelch-domains, but no apparent homologue of the PP2B family. The catalytic subunits of PPPs associate with regulatory protein partners that target them to well defined cellular locations and modulate their activity. Recent studies of phosphatase partners and their interactions have directed attention again to functional dissection of plant PPP families, and highlight their intriguing roles in the regulation of metabolism, cell cycle and development, as well as their roles in light, stress and hormonal signalling.
\end{abstract}

\section{Protein phosphatase families}

Protein phosphorylation and dephosphorylation represent a major form of reversible post-translational modification that controls many regulatory circuits in eukaryotes by modulating the conformation, activity, localization and stability of substrate proteins [1]. Whereas eukaryotic protein kinases appear to be derived from a single ancestor [2], protein phosphatases are thought to be recruited from various origins [3,4]. According to their substrate specificity, protein phosphatases are classified into families of Ser/Thr [serine/ threonine-specific phosphoprotein phosphatase (PPP) and metal ion-dependent protein phosphatase (PPM)], Tyr [phosphotyrosine phosphatase (PTP)], and dual specificity phosphatase (DSP) Ser/Thr- and Tyr-specific enzymes (Figure 1). DSPs and PTPs use an active Cys residue to catalyse analogous hydrolytic reactions, and their catalytic pockets share common structural features. Ser and Thr sidechains are dephosphorylated in a bicentral metal ionassisted reaction by PPPs (which include subfamilies of PP1, PP2A and PP2B, and novel phosphatases) and by PPMs (also known as PP2Cs). Except for PTPs that play crucial roles in animal cell differentiation, plants seem to use members of all known phosphatase families $[5,6]$.

Corresponding author: Koncz, C. (koncz@mpiz-koeln.mpg.de).

Available online 23 March 2007.
This review focuses on the regulatory functions of the Arabidopsis PPP family of Ser/Thr phosphatases, including recent advances in functional studies of novel enzymes (PP4 to PP7 and PPP kelch phosphatases).

Catalytic subunits of Arabidopsis PPPs are encoded by 26 genes (Figure 2). PP2B (also known as calcineurin or PPP3), which is an important $\mathrm{Ca}^{2+}$-regulated PPP in other eukaryotes, is absent in plants. PPPs occur in various complexes with regulatory and targeting subunits, which modulate the activity and specificity of catalytic subunits, and target the enzymes to specific locations $[7,8]$. Recent progress in functional identification of phosphatase subunits and interacting factors has highlighted the central roles of plant PPPs in cellular signalling.

\section{Protein phosphatase 1}

Mammalian PP1s preferentially dephosphorylate the $\beta$ subunit of phosphorylase kinase and are specifically inhibited by the heat-stable inhibitors I-1 and I-2. By contrast, PP2As dephosphorylate the $\alpha$ subunit of phosphorylase kinase and are insensitive to I-1 and I-2. Okadaic acid is a more potent inhibitor of PP2A than of PP1. Based on these distinctive features, PP1 phosphatases were identified in several plant species [9]. Arabidopsis PP1 isolated by microcystin affinity chromatography is inhibited by okadaic acid and inhibitor I-2, but shows greater sensitivity to microcystin-LR, nodularin, and calyculin A compared with the mammalian PP1s. Mass spectrometry analysis of purified Arabidopsis PP1s revealed between four and six catalytic subunit isoforms [10], whereas heterologous probes identified eight TOPP (Type One Protein Phosphatase) genes [11,12]. TOPP7 / AtPP1bg has now been designated TOPP6 and At5g43380 has been renamed TOPP7. Sequencing the Arabidopsis genome revealed a ninth gene for a TOPP8 isoenzyme (PP1 iso8), which is also expressed [13]. Plant TOPP and animal PP1 sequences show significant phylogenetic divergence [14]. Because of the heterogeneity of their N- and C-terminal domains, TOPPs can also be classified into distinct subfamilies (Figure 2). All TOPPs are likely to be active enzymes because they carry all the conserved residues that make up the active site of rabbit PP1 $\alpha$ [15]. 


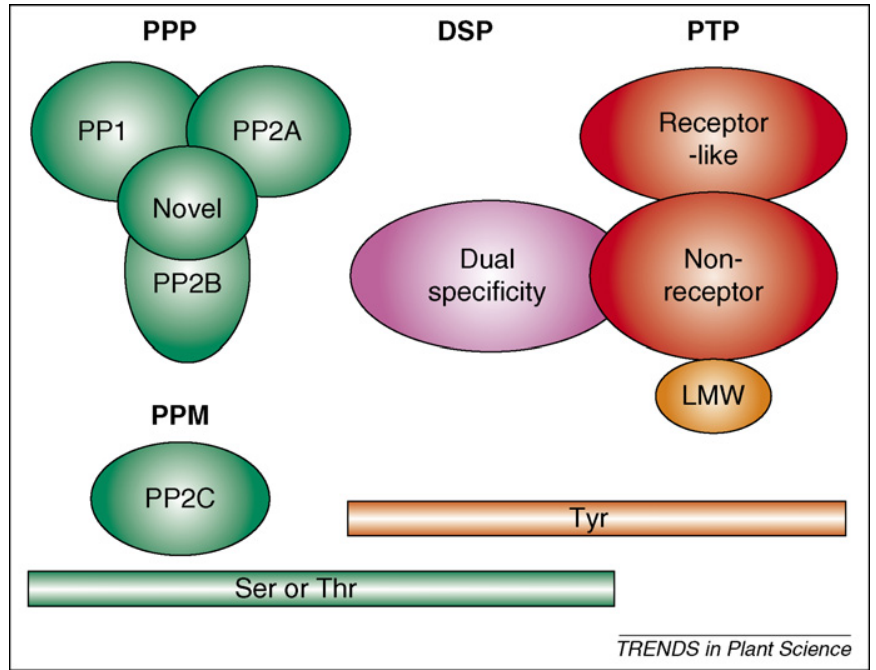

Figure 1. Classification of protein phosphatases according to their substrate specificities. Ser/Thr phosphatases include the phosphoprotein phosphatase PPP and metal ion-dependent PPM families. PPMs are often referred to as PP2Cs. The PPP group includes subfamilies of PP1 (known as TOPP in plants), PP2A, PP2B, and a novel class of phosphatases. PP2Bs are not present in plants. The phosphotyrosine phosphatase (PTP) family contains receptor-like, non-receptor, and low molecular weight (LMW) phosphatases. The catalytic mechanisms of PTPs are closely related to the dual specificity phosphatases (DSPs), which mediate the dephosphorylation of serine, threonine and tyrosine residues.

Several TOPPs are predicted to function in cell cycle regulation. TOPP3 and TOPP6 contain putative CDK2 recognition sites [16], but it is unknown whether they are inhibited by CDK2 kinases. TOPP2 suppresses the budding yeast glc7 mutation, which is not complemented by TOPP1 and TOPP3 [17]. TOPP1 is a suppressor of the fission yeast $c d c 25^{\text {ts }} /$ wee $1^{-}$mutation, which arrests the cell cycle before mitosis but, unlike TOPP2, it cannot suppress the defect of sister chromatid separation in the dis2-11 mutant [18,19]. TOPP6/AtPPbg complements the Aspergillus nidulans bimG11 mutation that, like dis2-11, causes a temperature-sensitive defect in anaphase [12].

TOPP isoforms are detected in large protein complexes and, hence, are likely to be able to bind several interacting proteins [10]. TOPP1 has been reported to show two-hybrid interaction with AXR3, a member of the AUX/IAA family of transcription factors, which suggests an involvement in auxin signalling (J. Jowett et al., unpublished) ${ }^{*}$. Arabidopsis encodes potential orthologues of some ancient PP1 regulatory subunits, including the inhibitor-2, inhibitor3 and nuclear SDS22 proteins (Table 1), which form multiple cooperative contacts with the catalytic subunit without the involvement of the conserved PP1-binding $\mathrm{RVxF}$ signature [20]. In addition, a putative orthologue of PP1 regulator NIPP-1 (At5g47790), which contains a PP1-binding motif, has also been identified [21]. We found that DDL (At3g20550) corresponds to another NIPP-1 orthologue that carries a forkhead-associated domain [22]. However, whether these NIPP-1-like proteins function as phosphatase regulators is an open question. A database report indicates that the $d d l$ mutant has delayed growth, curly leaves, and abnormal flowers, suggesting a

\footnotetext{
* J. Jowett et al. (2003) Identification and characterisation of AXR-3-interacting proteins (Poster 343), 14th International Conference on Arabidopsis Research, 20-24 June 2003, Madison, WI, USA.
}

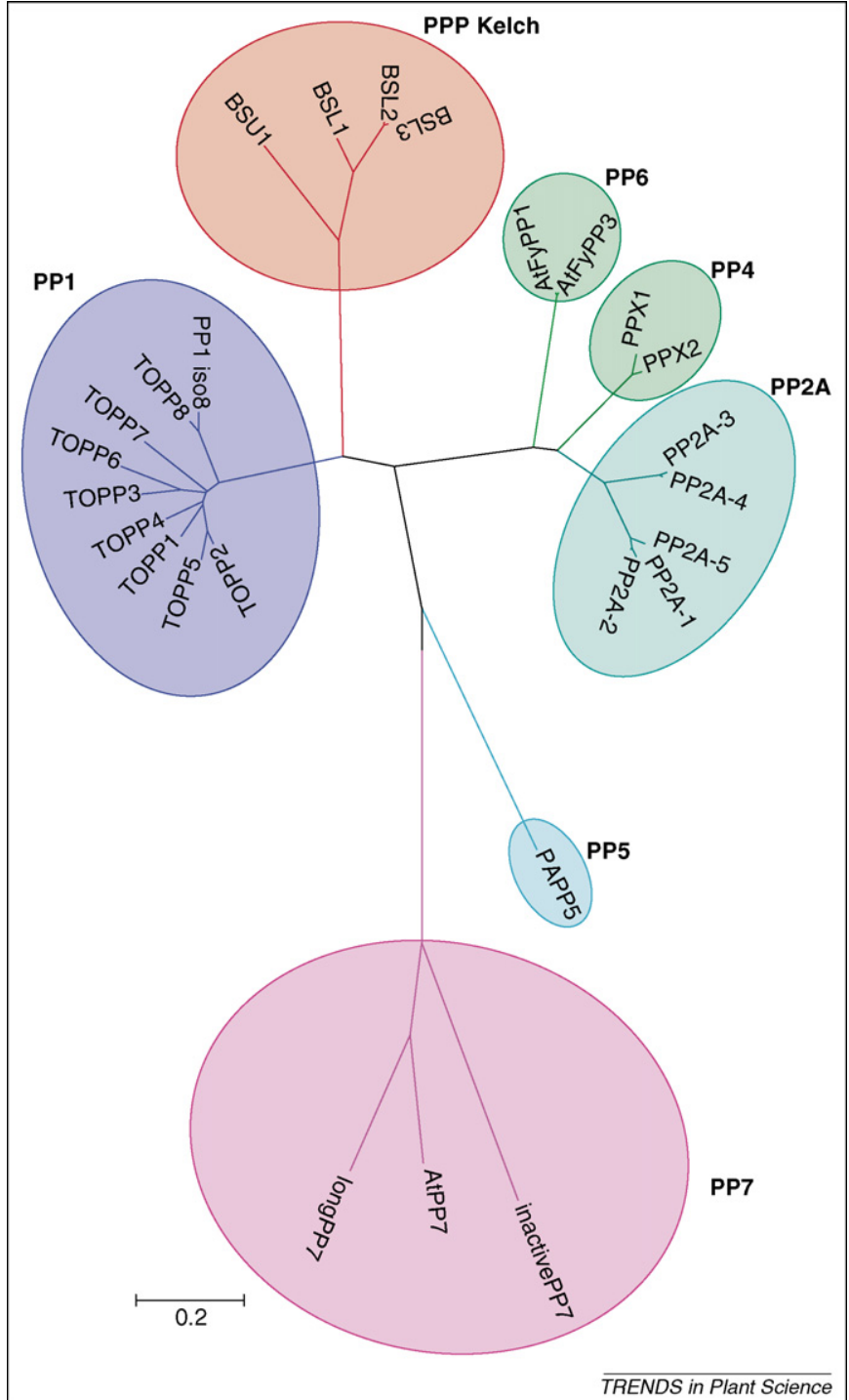

Figure 2. Family tree of Arabidopsis thaliana phosphoprotein phosphatase (PPP) catalytic subunits. Phylogenetic and molecular evolutionary analyses of amino acid sequences of PPP catalytic subunits were conducted using the MEGA software version 3.1 [64]. Subfamilies are circled and labelled with abbreviations explained in the text. The bar represents 0.2 amino acid substitutions per site in the primary structure. The AGI numbers of phosphatases are: TOPP1 $=$ At2g29400, TOPP2 = At5g59160, TOPP3 = At1g64040, TOPP4 = At2g39840, TOPP5 = At3g46820, TOPP6 $=$ At4g11240, TOPP7 = At5g43380, TOPP8 = At5g27840, PP1 iso8 = At3g05580, PP2A-1 $=$ At1g59830, PP2A-2 = At1g10430, PP2A-3 = At3g58500, PP2A-4 = At2g42500, PP2A-5 = At1g69960, PPX1 = At4g26720, PPX2 = At5g55260, PAPP5 = At2g42810, AtFyPP1 = At1g50370, AtFyPP3 = At3g19980, AtPP7 = At5g63870, longPP7 = At1g48120, inactivePP7 $=$ At5g10900, BSU1 $=$ At1g03445, BSL1 $=$ At4g03080 BSL2 = At1g08420, BSL3 = At2g27210.

role for DDL in regulating plant development (http:// arabidopsis.org).

\section{Protein phosphatase 2A}

Catalytic $(36 \mathrm{kDa})$ subunits of PP2A phosphatases occur either in association with a $65-\mathrm{kDa}$ regulatory $\mathrm{A}$ subunit, or together with a third variable B-subunit in heterotrimeric complexes [7]. The B subunits, which determine the substrate specificity and subcellular localization of PP2As, are classified into $55-\mathrm{kDa} \mathrm{B}, 54-74-\mathrm{kDa} \mathrm{B}^{\prime}$, and $72-130-$ $\mathrm{kDa} \mathrm{B}^{\prime}$ subunit families. PP2As have been reported to regulate the activity of key plant metabolic enzymes (e.g. sucrose phosphate synthase, 3-hydroxy-3-methylglutaryl-CoA reductase-1 and nitrate reductase) in vitro, but 
Table 1. Putative Arabidopsis thaliana PP1 regulator proteins

\begin{tabular}{|c|c|c|c|}
\hline AGI number & Name & $\begin{array}{lll}100 & 200 \\
1 & 100\end{array}$ & Domain $^{a}$ \\
\hline At5g47790 & NIPP-1 homologue & $-\infty$ & FHA \\
\hline At3g20550 & DDL (DAWDLE) & FHA. & $\mathrm{FHA}$ \\
\hline At5g19680 & SDS22 homologue & 중줏줒줒 & LRR RI \\
\hline At5g52200 & Inhibitor-2 homologue & & IPP-2 \\
\hline At2g31305 & Inhibitor-3 homologue & ES & PPI_Ypi1 \\
\hline
\end{tabular}

${ }^{a}$ Domains were identified using the Smart search program (http://smart.embl-heidelberg.de). Blue boxes represent unstructured elements, pink box shows a region of low complexity.

FHA: forkhead-associated domain, LRR: leucine rich repeat, IPP-2: protein phosphatase inhibitor-2 domain, PPI_Ypi1: Saccharomyces cerevisiae protein phosphatase 1 inhibitor Ypi1 domain. The scale shows the number of amino acid residues.

most of these data have yet to be verified in vivo [23,24]. Arabidopsis has five PP2A catalytic subunits, which are classified into two groups (Figure 2). All PP2A catalytic subunit genes are expressed in all organs, albeit at different levels [25]. Overexpression of putative G-protein coupled receptor GCR1 was found to stimulate expression of an unidentified PP2A catalytic subunit gene coinciding with the upregulation of gibberellin signalling [26]. The silencing of a subfamily of tomato PP2A catalytic subunit genes enhanced the response to bacterial and fungal avirulence proteins, increased PR gene expression, and localized cell death in stems and leaves, which suggests a potential role for PP2A in defence signalling [27].

Arabidopsis PP2A subunit A isoforms PDF1, PDF2 and RCN1 (Table 2) are composed of tandem HEAT repeats that form a hook-like architecture for binding the catalytic and regulatory B subunits [28]. RCN1 (roots curl in NPA) was first defined as a regulator of auxin transport and gravitropism [29]. In addition to increasing sensitivity to naphthylphthalamic acid (NPA), the ren1 mutation results in abscisic acid (ABA) insensitivity at the levels of seed germination, guard cell responses and gene expression, reduces PP2A activity, and leads to defects in apical hook formation, as well as in root and hypocotyl elongation [30,31]. Remarkably, the eer 1 (enhanced ethylene response 1) mutant carries a deletion in the RCN1 gene. RCN1 interacts in vitro with the PP2A-1 catalytic subunit that can bind the Raf-like kinase CTR1, a negative regulator of ethylene signalling [32]. Although RCN1 and PP2A-1 are not phosphorylated by CTR1, it is possible that CTR 1 is a substrate of PP2A. RCN1 was also identified as a two-hybrid binding partner of cyclophilin ROC7. However, silencing ROC7, unlike the rcn1 mutation, stimulates root growth without affecting wild-type NPA response [33]. Plants carrying mutations in the PP2A subunit A genes PDF1 and PDF2 show only slight phenotypic changes [34]. However, double mutants carrying $r c n 1$ and either $p d f 1$ or $p d f 2$ exhibit severe deficiencies, including abnormal embryogenesis, defective radial cell expansion, dwarfing and sterility. This indicates that RCN1 plays a fundamental role in the regulation of PP2A activity. Because PP2A subunit A isoforms perform partially overlapping functions, the effects of $p d f 1$ and $p d f 2$ mutations are unmasked only when RCN1 is absent.

Little is known about the functions of the two Arabidopsis $\mathrm{PP} 2 \mathrm{~A} 55-\mathrm{kDa} \mathrm{B}$ subunit isoforms that carry five degenerate WD-40 repeats (Table 2) and show 43-48\% amino acid sequence identity with yeast and animal B subunits [35]. Arabidopsis has nine PP2A B' subunits, of which only $\mathrm{AtB}^{\prime} \alpha$ and $\mathrm{AtB}^{\prime} \beta$ contain putative nuclear targeting sequences. The $A t B^{\prime} \gamma$ gene produces alternatively spliced transcripts. One of these, carrying a $5^{\prime}$ intron, accumulates under heat stress, and the $5^{\prime}$-intron serves as a translational enhancer under normal and heat stress conditions [36]. Arabidopsis encodes five PP2B" subunits (Table 2) that carry putative calcium-binding EF-hand motifs. Furthermore, TON2 (TONNEAU2) represents a $\mathrm{B}^{\prime \prime}$-like subunit, which shows similarity to the human $\mathrm{B}^{\prime \prime}$-subunit PR72 and interacts with the PP2A catalytic subunit in two-hybrid assays. The ton 2 mutation affects seedling body organization and causes abnormalities in microtubule assembly and in the formation of the preprophase band before mitosis [37].

In addition to conserved subunits, PP2A enzymes interact with various cellular and viral proteins. For example, yeast Tap42 can associate with PP2A and with Sit4, a closely related phosphatase and key enzyme of the TOR signalling pathway [38]. Arabidopsis TAP46 (46 kDa) represents a TAP42 orthologue that shows co-immunoprecipitation with a PP2A catalytic subunit. Transcription of TAP46 is induced by chilling, which suggests a role for a TAP46-PP2A complex in cold stress signalling [39].

\section{Protein phosphatase 4}

PP4 (also known as PPX) phosphatases are structurally related to the catalytic subunits of PP2A and are implicated in the regulation of microtubule-centrosome interactions in animals [40]. Arabidopsis encodes two PP4 enzymes, PPX-1 and PPX-2 (Figure 2), which are expressed at low levels in various organs. PPX-1 shows co-localization with ferredoxin in root epidermal cells, and immunolocalization indicates that PPX-1 is a lumenal protein in root plastids [41]. However, it is still unclear 
Table 2. Arabidopsis thaliana PP2A regulatory subunits

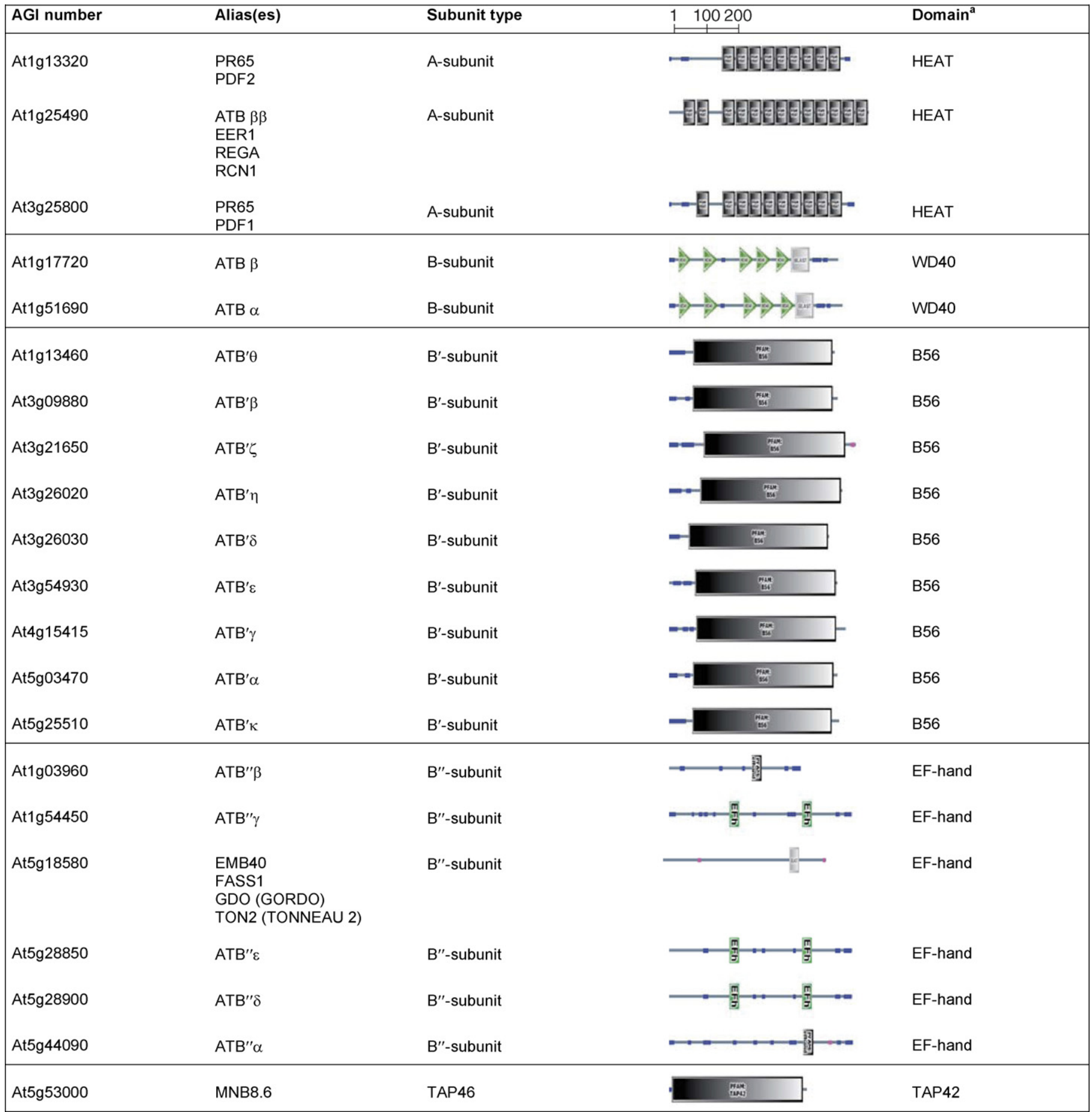

${ }^{a}$ Domains were identified with the Smart program (http://smart.embl-heidelberg.de). Blue boxes represent unstructured elements, pink boxes show regions of low complexity. BLAST means that the given domain was found only in a blast search. PFAM indicates that the PFAM collection of domains was used for identification. The scale shows the number of amino acid residues.

whether PPX-1 acts in the regulation of plastid-specific protein kinases and substrates.

\section{Protein phosphatase 5}

Although present in all eukaryotes, PP5s have not been extensively studied because they show low activity in vitro and little similarity to other phosphatases (Figure 2). PP5s carry three $\mathrm{N}$-terminal tetratricopeptide (TPR) motifs that, together with their C-terminal sequences, act as autoinhibitory domains. PP5s are activated by arachidonic acid and polyunsaturated fatty acids that bind to the TPR domains, and are inhibited by okadaic acid and microcystin. Mammalian PP5s are components of glucocorticoid receptor/Hsp90 and cryptochrome (CRY1 and CRY2) complexes and involved in the regulation of ion channels, hormone receptor signalling, cell cycle and apoptosis. Arachidonic acid and the microtubule depolymerizing agent nocodazol stimulate dissociation of PP5 from 
heat-shock protein complexes and its subsequent proteolytic activation $[42,43]$.

Like animal PP5s, plant PP5 enzymes are activated by arachidonic acid, unsaturated fatty acids and the removal of their TPR domains, and inhibited by okadaic acid [44]. In tomato and Arabidopsis, PP5 is encoded by single genes that produce two alternatively spliced transcripts encoding PP5 isoforms [45]. The 62-kDa isoform, which carries two $\mathrm{N}$-terminal trans-membrane domains, is localized in the endoplasmic reticulum and its function is as yet unknown. The 55-kDa PP5 isoform, PAPP5 (Phytochrome-Associated Protein Phosphatase 5; Figure 2), interacts with phytochrome PhyA in the two-hybrid system and preferentially dephosphorylates the biologically active far-red lightabsorbing Pfr form of oat PhyA in vitro [46]. The specificity of PAPP5 is probably due to preferential binding of its TPR domain to the Pfr form of PhyA. PAPP5 also binds PhyB; conversion of Pr to Pfr by red light-pulse enhances PAPP5 association with PhyB. Interaction of PAPP5 with PhyA is also spectral form dependent. In the dark, PAPP5 and PhyB are localized in the cytoplasm - upon illumination, they are translocated into the nucleus, where PAPP5 colocalizes with PhyB into speckles. Experiments with papp5 mutants and overexpressing lines show that increasing PAPP5 levels correlate with greater photoresponsiveness mediated by both PhyA and PhyB, which leads to increased expression of light-inducible genes. In the case of PhyA, dephosphorylation increases PhyA stability and affinity for a downstream signal transducer, the nucleoside diphosphate kinase 2 (NDPK2) [46-48]. PP5 also shows twohybrid interaction with the tomato I-2 disease resistance protein and two members of the Hsp90 family. Silencing of

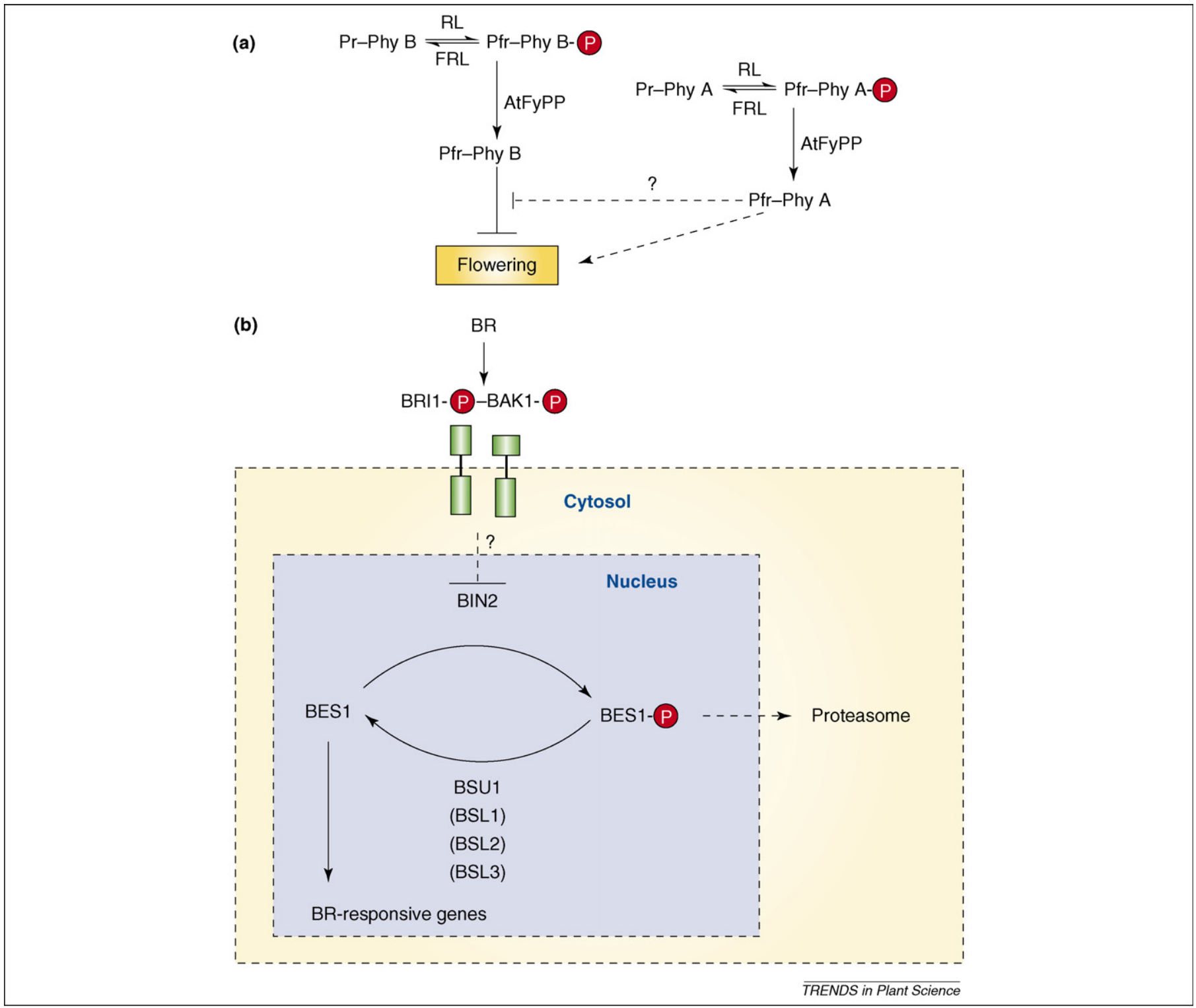

Figure 3. Physiological roles of plant PP6 and kelch-repeat containing phosphoprotein phosphatases. (a) PP6 phosphatases (AtFyPP1 and/or AtFyPP3) regulate flowering time by dephosphorylating phytochrome A and B. Abbreviations: AtFyPP, Arabidopsis thaliana phytochrome-associated protein phosphatase; FRL, far-red light; Pfr, active far-red absorbing form of phytochrome; Phy, phytochrome; Pr, inactive red-light absorbing form of phytochrome; RL, red light. $\mathrm{P}$ indicates the phosphorylated forms of the proteins. (b) Kelch-repeat-containing phosphatases are positive regulators of brassinosteroid signal transduction. Hypophosphorylated BES1 promotes the transcription of a set of brassinosteroid-responsive genes. Abbreviations: BR, brassinosteroids; BRI1 (BRASSINOSTEROID INSENSITIVE 1) and BAK1 (BRI1-ASSOCIATED RECEPTOR KINASE 1), plasma-membrane localized receptor kinases; BIN2 (BR-INSENSITIVE 2), glycogen synthase kinase-3; BES1 (bri1-EMS-suppressor 1), transcription factor. 
Hsp90, but not PP5, blocks cell death triggered by I-2, indicating that proper folding of I-2 is controlled by an Hsp90-PP5 complex [49].

\section{Protein phosphatase 6}

PP6 phosphatases, including Saccharomyces cerevisiae Sit4, Schizosaccharomyces pombe Ppe1, Drosophila melanogaster PPV and human PP6, are involved in the regulation of transcription, translation, morphogenesis and cell cycle [3]. Pea PP6, named FyPP, was found to interact with oat phytochrome PhyA and Arabidopsis PhyB in yeast. In vitro pull-down assays indicate that FyPP preferentially associates to the phosphorylated form of PhyA and Pfr forms of PhyA and PhyB [50]. Whereas PhyA and PhyB are imported to the nucleus upon red-light illumination, FyPP shows constitutive cytoplasmic localization. Arabidopsis has two PP6 orthologues, AtFyPP3 and AtFyPP1 (Figure 2), which differ at only two amino acid residues. Pea FyPP and AtFyPPs dephosphorylate autophosphorylated oat PhyA in vitro but, unlike PP2As, only in the presence of $\mathrm{Fe}^{2+}$ or $\mathrm{Zn}^{2+}$. Overexpression of pea $F y P P$ in Arabidopsis results in delayed flowering, whereas downregulation of $A t F y P P$ causes early flowering, suggesting phenotypic analogies with $p h y A$ and phyB mutants [50]. FyPP-mediated dephosphorylation of PhyA and PhyB has been proposed to have opposite effects on flowering time regulation (Figure 3a). The photoperiod-dependent flowering time regulators CONSTANS $(C O)$ and FLOWERING LOCUS T $(F T)$ show altered regulation in $F y P P$ antisense and atfypp 3 mutant lines. However, in the FyPP overexpressing lines, the $A t F y P P$ mRNA levels were unaffected and the atfypp 3 mutation alone also caused early flowering. This discrepancy was explained by the fact that $A t F y P P 3$ is expressed at higher levels under long-day conditions than under short days, whereas AtFyPP1 does not show such differential regulation [50]. Hence, the available data suggest that the Arabidopsis PP6 genes play distinct roles in PhyA and PhyB regulation and possibly in flowering time control.

\section{Protein phosphatase 7}

Plant PP7s have no close homologues in other kingdoms. In addition to AtPP7, Arabidopsis has two PP7-like genes (Figure 2). At1g48120 encodes a long PP7, whereas the product of At5g10900 is presumably inactive given that it lacks essential amino acids in the catalytic centre [15]. Unlike other PPPs, AtPP7 carries three inserts in the Cterminal half of its catalytic domain. AtPP7 can be activated by proteolytic cleavage, indicating that the first insert functions as an autoinhibitory region. AtPP7 is resistant to okadaic acid and calyculin but is inhibited by inorganic phosphate. Therefore, AtPP7 could possess a phosphate-binding site for allosteric regulation that could function as a phosphate sensory module [51]. AtPP7 also carries putative $\mathrm{Ca}^{2+}$-binding $\mathrm{EF}$ hand motifs and interacts with calmodulin in a $\mathrm{Ca}^{2+}$-dependent manner analogously to PP2B/calcineurin-like enzymes. However, unlike calcineurins, AtPP7 is inhibited by calmodulin. The activity of recombinant AtPP7 is reversibly regulated by redox-agents, but the biological significance of this observation is not yet clear [52].
Silencing of Arabidopsis AtPP7 was found to cause a defect in cryptochrome (CRY)-mediated blue-light signalling leading to a loss of hypocotyl growth inhibition by blue light and attenuation of blue-light-regulated gene expression $[53,54]$. AtPP7 appeared to be a potential candidate for a CRY phosphatase because cryptochromes are localized in the nucleus in darkness and AtPP7 also shows predominant nuclear localization [55]. However, no direct interaction was detected between AtPP7 and CRY1 [53]. Therefore, it was proposed that AtPP7 acts downstream of cryptochrome. Cryptochromes regulate the expression of chloroplast genes, such as $p s b D$, through blue-lightregulated nuclear $\sigma$ factors such as SIG5 [56]. AtPP7 could act through such a regulatory circuit given that SIG5 transcription is reduced in AtPP7 antisense plants but induced in wild type upon blue-light irradiation. Nonetheless, wild type and AtPP7-deficient plants show no significant difference in $C R Y 1$ and $C R Y 2$ transcription in blue or white light, and AtPP7 overexpression does not lead to blue-light hypersensitivity. Thus, further studies are required to define the precise function of AtPP7 in blue-light signalling.

\section{Protein phosphatases with kelch-repeat domains}

Biochemical and genetic studies have identified four novel phosphatases in Arabidopsis that are distantly related to PP1 and carry tandem N-terminal kelch-repeats that form $\beta$-propeller structures for protein-protein interactions [57,58] (Figure 2). A genetic screen for dominant suppressors of the brassinosteroid receptor bri1 mutation led to the identification of the bsu1-1D mutant, which overexpresses BSU1 (At1g03445), one of these kelch-repeat phosphatases [58]. The three other BSU1-like genes were designated BSL1 (At4g03080), BSL2 (At1g08420) and BSL3 (At2g27210).

BSU1 expressed in $E$. coli functions as an active phosphatase. Like PP1, BSU1 is inhibited only by okadaic acid but is insensitive to the PP1 inhibitor protein I-2. BSU1 was localized in the nucleus. BSU1 overexpression resulting from the dominant bsu1-1D mutation partially suppresses the dwarf phenotype of the bin2 mutant, which overexpresses a glycogen synthase kinase-3 homologue that acts as a negative regulator of brassinosteroid (BR) signalling [58]. Perception of the steroid hormone brassinolide by the LRR (leucine-rich receptor) kinase BRI1 triggers dephosphorylation and subsequent accumulation of transcription factors BES1 and BZR1 that function as positive regulators of BR-induced genes [59]. BIN2 acts downstream of BRI1 and by phosphorylation triggers ubiquitination and proteasomal degradation of BES1 [60-62] (Figure 3b). Dephosphorylated BES1 is more abundant in the bsu1-1D mutant, and BIN2-phosphorylated BES1 is dephosphorylated by recombinant BSU1 in vitro. Thus, BSU1 opposes the effect of BIN2 by promoting dephosphorylation and activation of BES1. In BR-stimulated cells, BIN2 is inhibited and BSU1 is activated by an unknown mechanism [63]. Combinations of bsu1 and bsl1 knockouts confer no BR-related phenotypic change, which indicates a functional overlap between the BSU1 and BSL phosphatases. However, bsl2 bsl3 double RNAi knock-down plants have a dwarf phenotype that resembles 
the phenotype of plants with weak bri1 alleles. Because the $B S L$ genes are expressed at higher levels than BSU1, BSL2 and BSL3 probably account for most of the BES1 dephosphorylating activity [58].

\section{Concluding remarks}

Despite of some unexplored fields, recent advances in the functional analysis of PPPs in Arabidopsis and other plants show that nearly all conserved families of protein phosphatases play pivotal roles in plant cell signalling. Studies of PPPs have shed light on numerous novel mechanisms, but many more regulatory interactions are probably still hidden. Without uncovering these, we shall not be able to determine how PPPs balance the action of $>1000$ protein kinases. The 'golden age' of plant PPPs is still to come, possibly as part of systems biology.

\section{Acknowledgements}

Our work was supported by the following grants: OTKA T038324 from the Hungarian Science Research Fund awarded to I.F. and the Deutsche Forschungsgemeinschaft SPP635 grant awarded to C.K. We acknowledge the participation of Éva Cseh and László Ökrész in the early phases of the project. We thank Pál Gergely for his advice and support.

\section{References}

1 Hunter, T. (1995) Protein kinases and phosphatases: the yin and yang of protein phosphorylation and signaling. Cell 80, 225-236

2 Manning, G. et al. (2002) Evolution of protein kinase signaling from yeast to man. Trends Biochem. Sci. 27, 514-520

3 Cohen, P.T.W. (1997) Novel protein serine/threonine phosphatases: variety is the spice of life. Trends Biochem. Sci. 22, 245-251

4 Denu, J.M. and Dixon, J.E. (1998) Protein tyrosine phosphatases: mechanisms of catalysis and regulation. Curr. Opin. Chem. Biol. 2, 633-641

5 Kerk, D. et al. (2002) The complement of protein phosphatase catalytic subunits encoded in the genome of Arabidopsis. Plant Physiol. 129, 908-925

6 Schweighofer, A. et al. (2004) Plant PP2C phosphatases: emerging functions in stress signaling. Trends Plant Sci. 9, 236-243

7 Janssens, V. and Goris, J. (2001) Protein phosphatase 2A: a highly regulated family of serine/threonine phosphatases implicated in cell growth and signalling. Biochem. J. 353, 417-439

8 Ceulemans, H. and Bollen, M. (2004) Functional diversity of protein phosphatase-1, a cellular economizer and reset button. Physiol. Rev. 84, $1-39$

9 Luan, S. (2003) Protein phosphatases in plants. Annu. Rev. Plant Biol. $54,63-92$

10 Stubbs, M.D. et al. (2001) Purification and properties of Arabidopsis thaliana type 1 protein phosphatase (PP1). Biochim. Biophys. Acta $1550,52-63$

11 Lin, Q. et al. (1998) Molecular cloning and chromosomal mapping of type one serine/threonine protein phosphatases in Arabidopsis thaliana. Plant Mol. Biol. 37, 471-481

12 Arundhati, A. et al. (1995) A novel Arabidopsis type 1 protein phosphatase is highly expressed in male and female tissues and functionally complements a conditional cell cycle mutant of Aspergillus. Plant J. 7, 823-834

13 Yamada, K. et al. (2003) Empirical analysis of transcriptional activity in the Arabidopsis genome. Science 302, 842-846

14 Lin, Q. et al. (1999) Molecular evolution of type 1 serine/threonine protein phosphatases. Mol. Phylogenet. Evol. 12, 57-66

15 Goldberg, J. et al. (1995) Three-dimensional structure of the catalytic subunit of protein serine/threonine phosphatase-1. Nature 376, 745753

16 Kwon, Y.G. et al. (1997) Cell cycle-dependent phosphorylation of mammalian protein phosphatase 1 by cdc2 kinase. Proc. Natl. Acad. Sci. U. S. A. 94, 2168-2173

17 Smith, R.D. et al. (1995) Type-1 and type-2 protein phosphatases of higher plants. Adv. Protein Phosphatases 9, 105-120
18 Ferreira, P.C.G. et al. (1993) A protein phosphatase 1 from Arabidopsis thaliana restores temperature sensitivity of a Schizosaccharomyces pombe cdc $25^{\text {ts }} /$ wee $1^{-}$double mutant. Plant J. 4, 81-87

19 Nitscke, K. et al. (1992) Complementation of the cs dis2-11 cell cycle mutant of Schizosaccharomyces pombe by a protein phosphatase from Arabidopsis thaliana. EMBO J. 11, 1327-1333

20 Egloff, M.P. et al. (1997) Structural basis for the recognition of regulatory subunits by the catalytic subunit of protein phosphatase 1. EMBO J. 16, 1876-1887

21 Ceulemans, H. et al. (2002) Regulator-driven functional diversification of protein phosphatase 1 in eukaryotic evolution. Bioessays 24, 371381

22 Beullens, M. and Bollen, M. (2002) The protein phosphatase-1 regulator NIPP1 is also a splicing factor involved in a late step of spliceosome assembly. J. Biol. Chem. 277, 19855-19860

23 MacKintosh, C. (1992) Regulation of spinach leaf nitrate reductase by reversible phosphorylation. Biochim. Biophys. Acta 1137, 121-126

24 Douglas, P. et al. (1997) Three spinach leaf nitrate reductase-3hydroxy-3-methylglutaryl-CoA reductase kinases that are regulated by reversible phosphorylation and/or $\mathrm{Ca}^{2+}$ ions. Biochem. J. 325, 101109

25 Arino, J. et al. (1993) Protein phosphatases in higher plants: multiplicity of type 2A phosphatases in Arabidopsis thaliana. Plant Mol. Biol. 21, 475-485

26 Colucci, G. et al. (2002) GCR1, the putative Arabidopsis G proteincoupled receptor gene is cell cycle-regulated, and its overexpression abolishes seed dormancy and shortens time to flowering. Proc. Natl. Acad. Sci. U. S. A. 99, 4736-4741

$27 \mathrm{He}$ X. et al. (2004) Silencing of subfamily I of protein phosphatase 2A catalytic subunits results in activation of plant defense responses and localized cell death. Plant J. 38, 563-577

28 Groves, M.R. et al. (1999) The structure of the protein phosphatase 2A PR65/A subunit reveals the conformation of its 15 tandemly repeated HEAT motifs. Cell 96, 99-110

29 Garbers, C. et al. (1996) A mutation in protein phosphatase 2A regulatory subunit A affects auxin transport in Arabidopsis. EMBO J. $15,2115-2124$

30 Rashotte, A.M. et al. (2001) Genetic and chemical reductions in protein phosphatase activity alter auxin transport, gravity response, and lateral root growth. Plant Cell 13, 1683-1697

31 Kwak, J.M. et al. (2002) Disruption of a guard cell-expressed protein phosphatase 2A regulatory subunit, RCN1, confers abscisic acid insensitivity in Arabidopsis. Plant Cell 14, 2849-2861

32 Larsen, P.B. and Cancel, J.D. (2003) Enhanced ethylene responsiveness in the Arabidopsis eer1 mutant results from a lossof-function mutation in the protein phosphatase 2A A regulatory subunit, RCN1. Plant J. 34, 709-718

33 Jackson, K. and Söll, D. (1999) Mutations in a new Arabidopsis cyclophilin disrupt its interaction with protein phosphatase 2A. Mol. Gen. Genet. 262, 830-838

34 Zhou, H-W. et al. (2004) Disparate roles for the regulatory A subunit isoforms in Arabidopsis protein phosphatase 2A. Plant Cell 16, 709722

35 Corum, J.W. et al. (1996) Characterization of DNA sequences encoding a novel isoform of the $55 \mathrm{kDa} \mathrm{B}$ regulatory subunit of the type $2 \mathrm{~A}$ protein serine/threonine phosphatase of Arabidopsis thaliana. Plant Mol. Biol. 31, 419-427

36 White, R.J. et al. (2002) Functional analysis of the $\mathrm{B}^{\prime}$ subunit of Arabidopsis protein phosphatase type 2A. Plant Sci. 162, 201209

37 Camilleri, C. et al. (2002) The Arabidopsis TONNEAU2 gene encodes a putative novel protein phosphatase 2 A regulatory subunit essential for the control of the cortical cytoskeleton. Plant Cell 14, 833-845

38 Di Como, C.J. and Arndt, K.T. (1996) Nutrients, via the Tor proteins, stimulate the association of Tap42 with type $2 \mathrm{~A}$ phosphatases. Genes Dev. 10, 1904-1916

39 Harris, D.M. et al. (1999) The Arabidopsis homolog of yeast TAP42 and mammalian $\alpha 4$ binds to the catalytic subunit of protein phosphatase 2A and is induced by chilling. Plant Physiol. 121, 609-617

40 Brewis, N.D. et al. (1993) PPX, a novel protein serine threonine phosphatase localized to centrosomes. EMBO J. 12, 987-996

41 Pujol, G. et al. (2000) The Arabidopsis thaliana PPX/PP4 phosphatases: molecular cloning and structural organisation of genes and 
immunolocalization of the proteins to plastids. Plant Mol. Biol. 44, 499-511

42 Chinkers, M. (2001) Protein phosphatase 5 in signal transduction. Trends Endocrinol. Metab. 12, 28-32

43 Zeke, T. et al. (2005) Human protein phosphatase 5 dissociates from heat-shock proteins and is proteolytically activated in response to arachidonic acid and the microtubule-depolymerizing drug nocodazole. Biochem. J. 385, 45-56

44 Meek, S. et al. (1999) Microcystin affinity purification of plant protein phosphatases: PP1C, PP5 and a regulatory A-subunit of PP2A. FEBS Lett. 457, 494-498

45 de la Fuente van Bentem, S. et al. (2003) The subcellular localization of plant protein phosphatase 5 isoforms is determined by alternative splicing. Plant Physiol. 133, 702-712

46 Ryu, J.S. et al. (2005) Phytochrome-specific type 5 phosphatase controls light flux by enhancing phytochrome stability and affinity for a signal transducer. Cell 120, 395-406

47 Kim, J-I. et al. (2004) Phytochrome phosphorylation modulates light signaling by influencing protein-protein interaction. Plant Cell 16, 2629-2640

48 Shen, Y. et al. (2005) NDPK2 as a signal transducer in the phytochrome-mediated light signaling. J. Biol. Chem. 280, 5740-5749

49 de la Fuente van Bentem, S. et al. (2005) Heat shock protein 90 and its co-chaperone protein phosphatase 5 interact with distinct regions of the tomato I-2 disease resistance protein. Plant J. 43, 284-298

$50 \mathrm{Kim}, \mathrm{D}-\mathrm{H}$. et al. (2002) A phytochrome-associated protein phosphatase 2A modulates light signals in flowering time control in Arabidopsis. Plant Cell 14, 3043-3056

51 Kutuzov, M.A. and Andreeva, A.V. (2001) Noncompetitive inhibition of plant protein Ser/Thr phosphatase PP7 by phosphate. Biochem. Biophys. Res. Commun. 283, 93-96

52 Andreeva, A.V. et al. (2001) Purification of plant protein phosphatase PP7 and evidence for its redox regulation. Arch. Biochem. Biophys. 396 , 65-70
53 Moller, S.G. et al. (2003) PP7 is a positive regulator of blue light signaling in Arabidopsis. Plant Cell 15, 1111-1119

54 Ohgishi, M. et al. (2004) Functional analysis of each blue light receptor, cry1, cry2, phot1, and phot2, by using combinatorial multiple mutants in Arabidopsis. Proc. Natl. Acad. Sci. U. S. A. 101, 2223-2228

55 Andreeva, A.V. and Kutuzov, M.A. (2001) Nuclear localization of the plant protein Ser/Thr phosphatase PP7. Mol. Cell Biol. Res. Commun. $4,345-352$

56 Tsunoyama, Y. et al. (2004) Blue light-induced transcription of plastidencoded $p s b D$ gene is mediated by a nuclear-encoded transcription initiation factor, AtSig5. Proc. Natl. Acad. Sci. U. S. A. 101, 33043309

57 Kutuzov, M.A. and Andreeva, A.V. (2002) Protein Ser/Thr phosphatases with kelch-like repeat domains. Cell. Signal. 14, 745-750

58 Mora-Garcia, S. et al. (2004) Nuclear protein phosphatases with Kelchrepeat domains modulate the response to brassinosteroids in Arabidopsis. Genes Dev. 18, 448-460

59 Bishop, G.J. and Koncz, C. (2002) Brassinosteroids and plant steroid hormone signalling. Plant Cell 14 (Suppl.), S97-S110

60 He, J.X. et al. (2002) The GSK3-like kinase BIN2 phosphorylates and destabilizes BZR1, a positive regulator of the brassinosteroid signaling pathway in Arabidopsis. Proc. Natl. Acad. Sci. U. S. A. 99, 1018510190

61 Zhao, J. et al. (2002) Two putative BIN2 substrates are nuclear components of brassinosteroid signaling. Plant Physiol. 130, 12211229

62 Yin, Y. et al. (2005) A new class of transcription factors mediates brassinosteroid-regulated gene expression in Arabidopsis. Cell 120, 249-259

63 Vert, G. et al. (2005) A molecular mechanism of steroid hormone signaling in plants. Annu. Rev. Cell Dev. Biol. 21, 177-201

64 Kumar, S. et al. (2004) MEGA3: integrated software for molecular evolutionary genetics analysis and sequence alignment. Brief. Bioinform. 5, 150-163

\title{
Plant Science Conferences in 2007
}

\author{
21st Asian Pacific Weed Science Society Conference \\ 2-6 October 2007 \\ Colombo, Sri Lanka \\ http://www.apwss21.Ik/
}

XVI International Plant Protection Congress

15-18 October 2007

Glasgow, UK

http://www.bcpc.org/iapps2007/

\section{9th Conference of the International Society for Plant Anaerobiosis}

19-23 November 2007

Sendai, Japan

http://ispa-japan.miyakyo-u.ac.jp/

\author{
ASCB 47th Annual Meeting \\ 1-5 December 2007 \\ Washington, DC, USA \\ http://www.ascb.org/meetings/
}

